\title{
336 - Prevalence of cognitive frailty in a sample of Portuguese community-dwelling elderly people
}

Authors: Gaspar, P.M. , Facal, D. \& Juncos-Rabadán, O.

Background: Cognitive frailty (CF) has been considered as a subtype of frailty characterized by concurrent physical frailty and potentially cognitive impairment (Kelaiditi et al., 2013). Early detection of $\mathrm{CF}$ is an important issue in order to implement prevention and intervention to improve quality of life in aging. The aim of this study was to explore the prevalence of cognitive frailty in a sample of Portuguese old people, living in the community, and determine its relationship with sex, age, education and comorbidity.

Method: Two hundred forty nine community-dwelling participants aged over 60 years and without dementia and other neurological and psychiatric diseases were assessed to obtain sociodemographic, functional, health, physical, cognitive and socio-affective measures. Participants were classified with/without mild cognitive impairment using the Montreal cognitive test (MoCa Test), and as physically robust, pre-frail and frail using the criteria of Fried et al. (2001). A cognitive frailty classification variable was created with six groups that were compared on sociodemographic variables and on comorbidity measured with the Charlson Index Scale (ChIS).

Results: A $20.1 \%$ of participants were classified as physically robust and cognitively normal (Group 1), a $28.5 \%$ as physically pre-frail and cognitively normal (Group 2), a $11.2 \%$ as physically frail and cognitively normal (Group 3), a $6.8 \%$ as physically robust with cognitive impairment (Group 4), a $14.9 \%$ as physically pre-frail with cognitive impairment (Group 5 ), and a $18.5 \%$ as physically frail with cognitive impairment (Group 6). No significant group differences were registered in the distribution by sex. Significant differences were observed between Group 6 and Group 1 and Group 2 in age [ $F(5$, $243)=6.20, p<.01]$ and in education $[F(5,243)=6.54, p<.01]$, being the first older and with lower education level than the two last ones. Regarding comorbidity, Group 6 had significant higher scores in the ChIS $[F(5,243)=9.91, p<.01]$ than all the other groups

Conclusion: The study reveals that a $33.4 \%$ of the older adults and with less education level living in community suffer the two more advanced stages of cognitive frailty. Prevention and interventions measures are need to improve their quality of life.

References: Charlson M. Pompei P, Ales KL, McKenzie CRA, new method 01 classifying prognostic comorbidity in longitudinal studies: development and validation. J Chron Ois 1987: 40:

373-83.

Kelaiditi, E., Cesari, M., Canevelli, M., Van Kan, G. A., Ousset, P. J., Gillette-Guyonnet, S., et al. (2013). Cognitive frailty: rational and definition from an (IANA/IAGG) international consensus group. The journal of nutrition, health \& aging, 17(9), 726-734. doi: 10.1007/s12603-013-0367-2

Pereiro, A. X., Ramos-Lema, S., Lojo-Seoane, C., Guárdia-Olmos, J., Facal-Mayo, D., and Juncos-Rabadán, O. (2017). Normative data for the Montreal Cognitive Assessment (MOCA) in a Spanish sample of community-dweller adults. European geriatric medicine, 8(3), 240-244 Fried, L. P., Tangen, C. M., Walston, J., Newman, A. B., Hirsch, C., Gottdiener, J., ... \& McBurnie, M. A. (2001). Frailty in older adults: evidence for a phenotype. The Journals of Gerontology Series A: Biological Sciences and Medical Sciences, 56(3), M146-M157.

Key Words: Cognitive, Frail, Phisically 